\title{
Visualization of the equilibrium FCC catalyst surface by AFM and SEM-EDS
}

\author{
Oguz Bayraktar ${ }^{\mathrm{a}, *}$ and Edwin L. Kugler ${ }^{\mathrm{b}}$ \\ ${ }^{a}$ Department of Chemical Engineering, Izmir Institute of Technology, Gülbahçe Köyü, 35437, Urla-Izmir, Turkey \\ ${ }^{\mathrm{b}}$ Department of Chemical Engineering, CEMR, P.O. Box 6102, West Virginia University, Morgantown, WV 26506-6102, USA
}

Received 8 April 2003; accepted 17 July 2003

\begin{abstract}
The deposition of metal contaminants (e.g., Ni, V, and Fe) from the hydrocarbon feed causes the deactivation of fluid catalytic cracking (FCC) catalyst used in petroleum refining. It is very important to understand the changes in the morphology and chemical composition on the catalyst surface and how these structural and chemical changes affect the catalyst performance. In this research, metal-contaminated FCC catalysts from a commercial unit have been characterized using AFM together with SEM-EDS. The AFM images showed the surface pores as well as the features that surround the pore's entrance on the catalyst surface. Catalyst surface contains debris that appear as bright spots in AFM images. SEM-EDS results have shown the presence of iron in these bright spots. Fe enrichment at the catalyst particle surface was also confirmed by XPS analyses.
\end{abstract}

KEY WORDS: AFM; SEM-EDS; FCC catalysts; metal contamination.

\section{Introduction}

A common problem in petroleum refining is catalyst deactivation due to the deposition of metal contaminants (e.g., nickel, vanadium, and iron) on the catalyst from the hydrocarbon feed. Catalyst activity decreases as the production of coke and hydrogen increase at the expense of gasoline [1]. The presence of metal contaminants on the FCC (fluid catalytic cracking) catalyst has been widely studied by using microscopic and elemental imaging techniques such as scanning electron microscopy (SEM) [2,3] and secondary ion mass spectrometry (SIMS) [4,5]. These results have shown that vanadium is mobile and accumulates throughout the catalyst particles with a preference for rare earthexchanged Y-zeolite. On the other hand, nickel and iron tend to remain fixed at the catalyst particle surface where they are deposited.

Significant effects on catalyst performance are often observed by slightly modifying the catalyst composition and structure, especially on the surface. This finding is not surprising since the hydrocarbon feed for cracking reactions first contacts the catalyst surface before it diffuses into the catalyst pores. Assuming that cracking reactions take place mainly on the top $10-15 \mu \mathrm{m}$ of the FCC catalyst, novel techniques for characterizing surface chemical composition and pore structure become extremely important for understanding the catalyst performance. An example of the impact of new

\footnotetext{
* To whom correspondence should be addressed.

E-mail: oguzbayraktar@iyte.edu.tr
}

instrumental techniques is provided by atomic force microscopy (AFM), a powerful technique that can generate the images of surfaces with atomic resolution. AFM has been used successfully to investigate the surface features of pillared rectorite catalysts $[6,7,8]$. Several studies were also conducted to observe the FCC catalyst surfaces using AFM $[9,10]$. In these studies, both FCC catalysts artificially contaminated with vanadyl naphthenenate and commercial equilibrium FCC catalysts with metal contaminants have been used. Their AFM images revealed a surface architecture characterized by valleys, ridges, crevices, and slits [9]. They have concluded that vanadium oxides decrease the surface roughness by filling valleys and cracks. In the case of equilibrium FCC catalysts, AFM images have shown that the catalyst surface contains debris. It has been speculated that this debris might be nickel and vanadium oxides [10].

In this work, actual surface images of the equilibrium FCC catalyst samples with different metal contamination levels have been obtained to study the details of the surface topography using AFM. Although AFM provides us with the actual images of the catalyst surfaces, unfortunately it does not give any information on surface chemical composition that may give information on the identity of the above-mentioned debris. To the best of our knowledge, nobody in the literature has identified the nature of the debris observed on the surface of the FCC catalyst. This study aims at further investigating the nature of the debris observed in the AFM images of commercial equilibrium FCC catalysts. 


\section{Experimental}

\subsection{Catalysts}

Commercial equilibrium FCC catalysts were supplied by Ashland, Inc. The calcined equilibrium FCC catalysts were labeled ECat-LOW, ECat-INT, and ECat-HIGH on the basis of their contaminant-metals concentration. Characterization data on these equilibrium catalysts are provided in table 1 .

\subsection{Atomic force microscopy (AFM)}

The FCC catalyst particles were fixed on a sticky carbon tape. The AFM used in this work was an AutoProbe $^{\mathrm{TM}}$. CP (Park Scientific Instruments, now merged with TopoMetrix into ThermoMicroscopes) operating in contact mode. It has the combined capabilities of an AFM and STM (scanning tunneling microscope) and is generically called a scanning probe microscope (SPM). This unit is an in-air (or ex situ) instrument that is placed on a concrete slab suspended by flexible cords to provide isolation from external mechanical vibrations. AFM was calibrated using gold grating before the actual AFM scans.

\subsection{Scanning electron microscopy-energy dispersive $X$ - ray spectroscopy (SEM-EDS)}

SEM-EDS analyses were performed at Image and Chemical Analysis Laboratory (ICAL), Montana State University and at Materials Research Laboratories (MRL), Inc., Struthers, OH.

\section{Results and discussion}

Figure 1(A), (B), and (C) show the three-dimensional AFM images for calcined ECat-LOW, ECat-INT, and ECat-HIGH, respectively. These AFM images show the surface pores as well as the features that surround the pore's entrance on the catalyst surface. In all of these images, the catalyst surface contains debris that appear as bright spots in the images. As mentioned earlier,
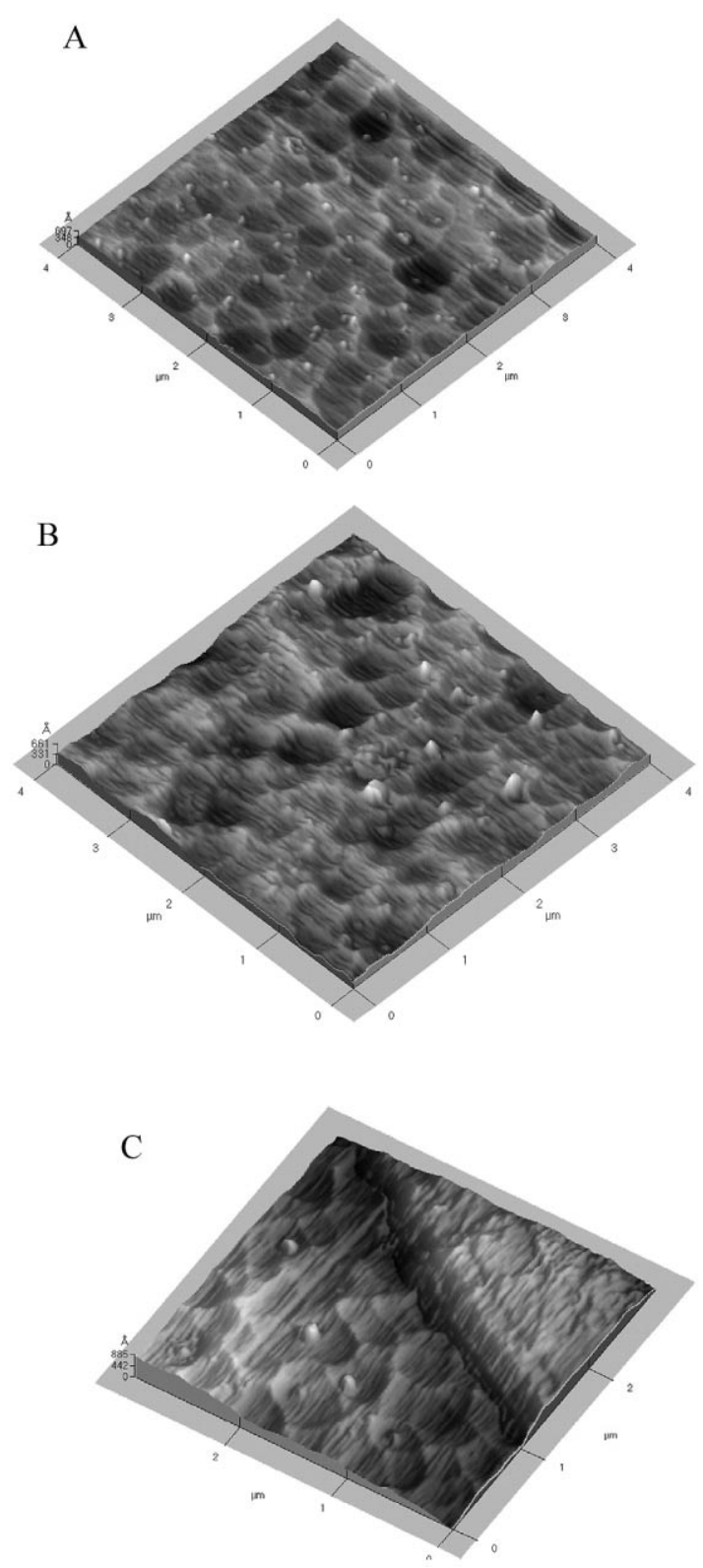

Figure 1. 3-D AFM images of the equilibrium FCC catalysts: (A) ECat-LOW, (B) ECat-INT, and (C) ECat-HIGH.

other researchers [10] have also made similar observations. However, the exact chemical composition of these debris has not been given in the literature. From these

Table 1

Nominal properties of equilibrium catalysts from Ashland Inc.

\begin{tabular}{lccc}
\hline Catalyst & ECat-LOW & ECAT-INT & ECat-HIGH \\
\hline BET surface area $\left(\mathrm{m}^{2} / \mathrm{g}\right)$ & 178 & 160 & 115 \\
Matrix surface area $\left(\mathrm{m}^{2} / \mathrm{g}\right)$ & 63 & 63 & 45 \\
Zeolite surface area $\left(\mathrm{m}^{2} / \mathrm{g}\right)$ & 115 & 97 & 70 \\
Metals $(\mathrm{ppm})$ & & & \\
Nickel & 300 & 900 & 2600 \\
Vanadium & 700 & 1700 & 6700 \\
Iron & 4400 & 6300 & 7000 \\
Microactivity & 71 & 69 & 62 \\
\hline
\end{tabular}


AFM images shown in figure 1, one can easily see the sizes of these debris and their locations. Another interesting observation made was the number of these debris and their organization on the catalyst surface. For ECat-LOW, this debris seems to be very small in size and it is distributed almost homogeneously in a wide surface range. For ECat-INT and ECat-HIGH, the size of this debris is slightly larger than that of ECat-LOW and it is randomly distributed on the catalyst surface. The metal concentration on the catalyst gives an idea about the age of the catalyst. The higher the metal concentration, the older the catalyst is. In this case, the metal concentration indicates that ECat-HIGH is the oldest of all. The increasing size of this debris with increasing metal concentrations shows that this debris has a tendency to agglomerate randomly as the catalyst ages. The number of this debris becomes smaller with increasing catalyst age. In the case of ECat-HIGH, surface cracks were observed on the catalyst surface (see figure 1(C)). These cracks can be attributed to the age of catalyst. Occelli et al. [9] have also observed similar type of cracks on the surface of FCC catalysts.

Figure 2 shows the SEM secondary electron micrographs of ECat-LOW (figure 2(A), (B), and (C)) and ECat-HIGH (figure 2(D), (E), and (F)) at different magnifications. The presence of this surface debris observed in AFM images was further confirmed by observing the similar type of features in SEM pictures given in figure 2 . The changes in the surface characteristics of equilibrium catalysts with increasing metal concentration can easily be distinguished by looking at figure 2(A) and (D). The SEM picture (figure 2(D)) of

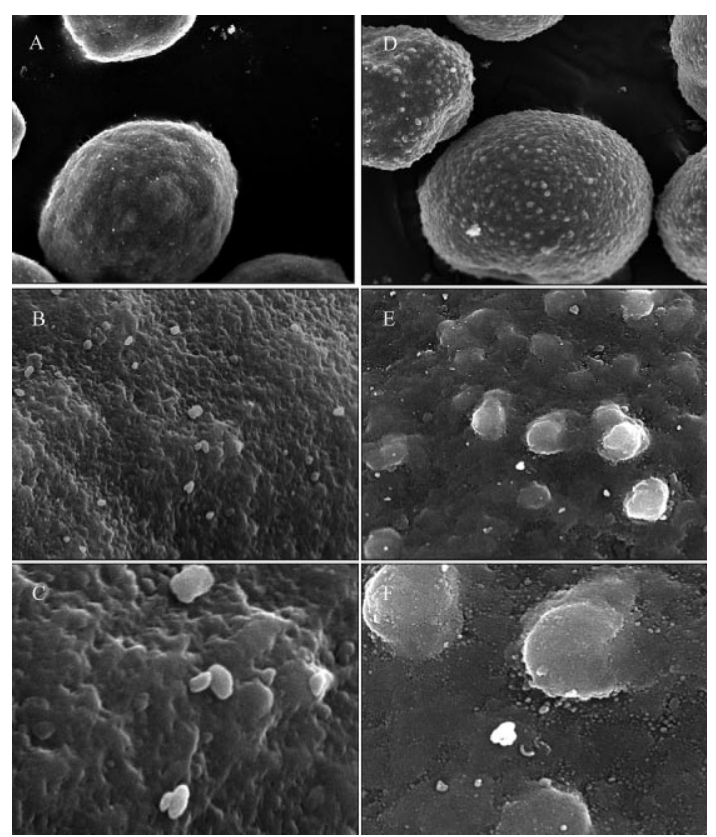

Figure 2. SEM micrographs of equilibrium FCC catalysts: (a), (b), and $(\mathrm{C})$ are for ECat-LOW at $\times 600, \times 5000$, and $\times 15000$ magnifications, respectively (left); (D), (E), and (F) are for ECat-HIGH at $\times 600$, $\times 5000$, and $\times 15000$ magnifications, respectively (right). highly contaminated equilibrium catalyst shows a puckered catalyst surface.

The distribution of contaminant metals on the equilibrium FCC catalyst surfaces was observed by scanning electron microscopy (SEM) in combination with X-ray energy dispersive spectroscopy (SEM-EDS). Elemental distribution maps were obtained to show the distribution on the sample of an element of interest. More dots or brighter spots indicate more of the element. The elemental distribution shown on the micrograph (distribution map) can then be related to the sample topography shown in a SEM secondary electron micrograph taken of the same area. Figure 3(A), (B), and (C) shows the secondary electron image, backscattered electron image, and the distribution map
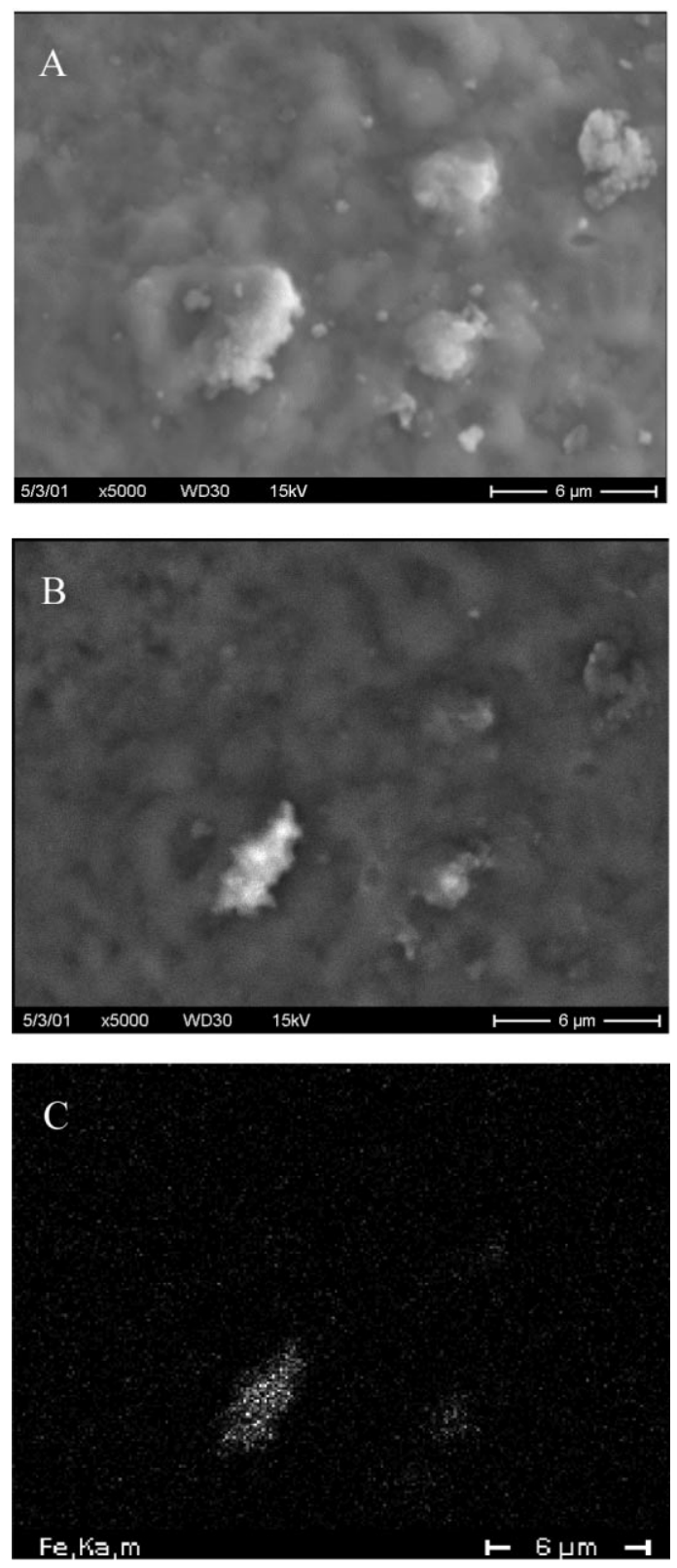

Figure 3. SEM images of ECat-HIGH: (A) secondary electron image, (B) backscattered electron image, and (C) distribution map of iron. 
of iron on the surface of ECat-HIGH, respectively. In a secondary electron image, large particulates as well as very small ones were observed on the catalyst surface. The small particulates may come together in the FCC process conditions and make larger ones, which are responsible for the formation of nodules on the catalyst surface. Several spots that varied in composition from the bulk were observed using the backscattered detector, which can detect the variations in density (figure 3(B)). Figure $3(\mathrm{C})$ shows the presence of iron in these large particulates. However, very small bright spots shown in figure 3(A) did not give a significant signal in the elemental distribution maps. This might be related to the low sensitivity of X-ray EDS technique at very low elemental concentrations. Most of the elemental distribution maps of catalyst surfaces have shown that $\mathrm{Fe}$ exists in the aggregates that appear as bright spots on the elemental distribution maps. In addition to surface scans with SEM-EDS, spot SEM-EDS analyses were also performed (results are not shown). Using spot SEM-EDS analysis option enhances the sensitivity of this technique slightly. In spot SEM-EDS analysis, the catalyst particle image was first obtained at the computer screen. Then the points of interest were

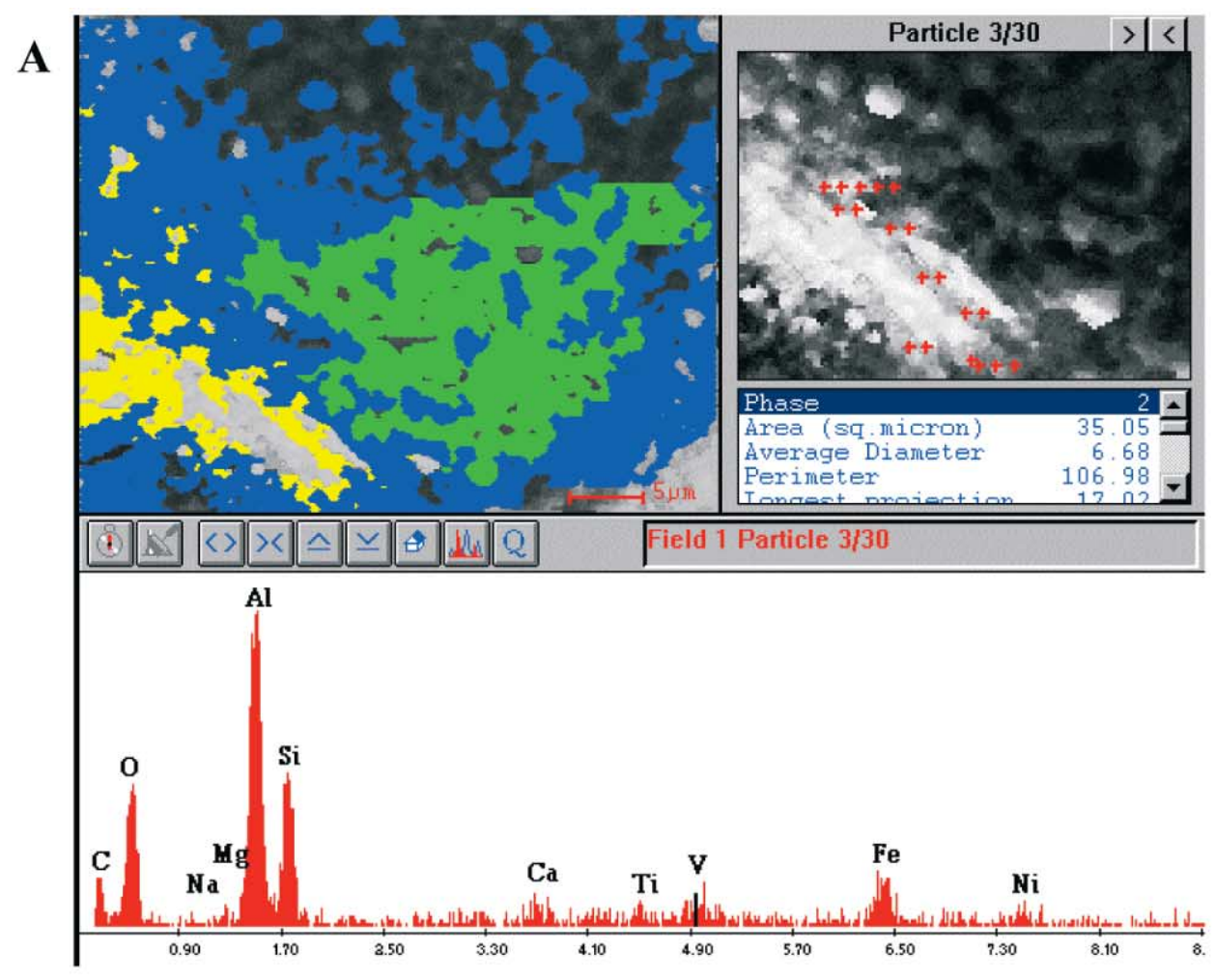

B

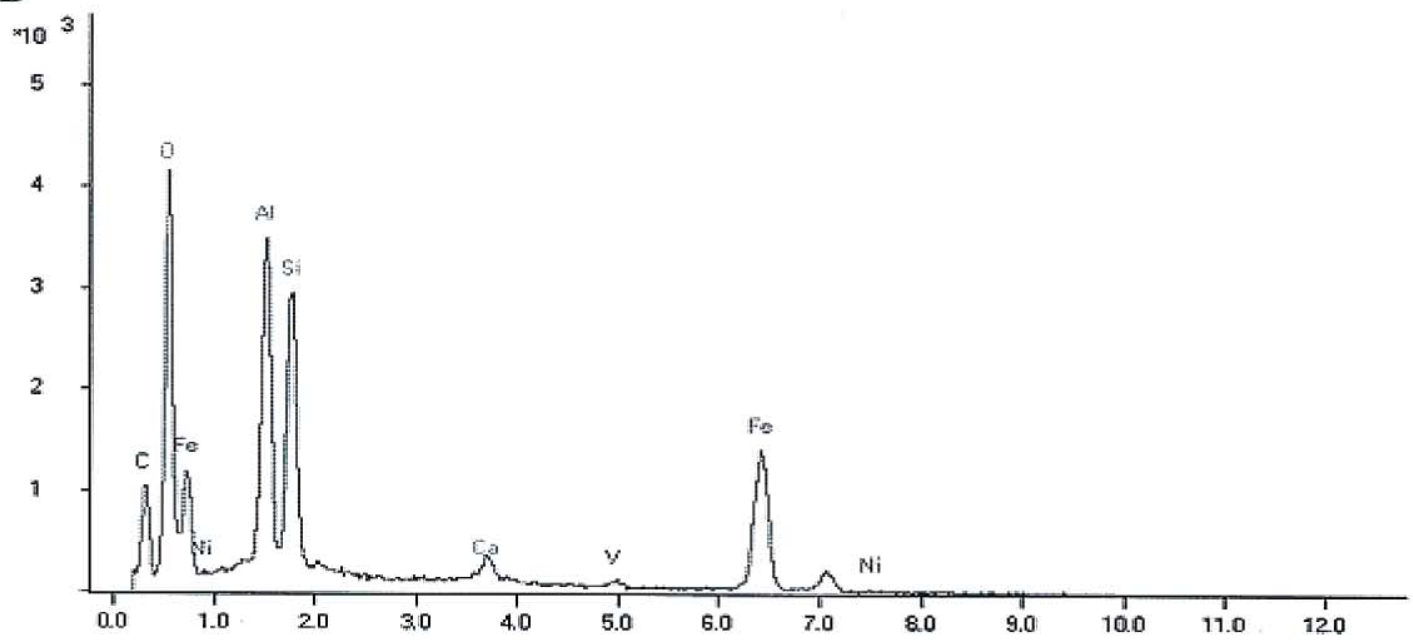

Figure 4. (A) SEM-EDS image and spectrum of ECat-HIGH and (B) EDS spectrum of a typical bright spot on the surface of ECat-HIGH catalyst particle. 
selected and the analysis was performed on each of them. By selecting points at areas with different morphology, it was possible to explore the metal content of these selected spots. In order to identify this debris, more than 15 locations were marked on a single catalyst particle. These locations were analyzed for possible elements. These results have shown the presence of iron and titanium at these marked bright locations on the catalyst surface. Typical EDS spectra are given in figure 4(a) and (b). As seen from these spectra, bright spots on the catalyst surface contain iron. This is not surprising since iron (mostly $\mathrm{Fe}_{2} \mathrm{O}_{3}$ ) and titania (mainly rutile) are the impurities associated with clay (kaolin) used during the manufacturing process of commercial FCC catalysts. Titanium detected by SEM-EDS analysis presents in the catalyst structure itself at a concentration of $0-3 \mathrm{wt} \%$. The presence of iron in the parent materials further complicates the observations of increases in iron content on these equilibrium catalysts. The iron deposited on the catalyst may come from the feed, from corrosion scale, or from the equipment degradation as a result of the feed containing high naphthenic acid. In order to compare the surface and bulk concentrations of metals present on the equilibrium catalyst samples, X-ray photoelectron spectroscopy (XPS) analysis was performed on catalyst particle surfaces. XPS is an analytical technique for the study of thin material layers that uses X-rays to stimulate desorption of electrons in the near surface region (2$4 \mathrm{~nm}$ ) of a specimen. The surface enrichment ratio for any element can quantitatively be measured by dividing its surface concentration determined from XPS data by its bulk concentration obtained from inductively coupled plasma (ICP) analysis. The results from XPS analyses have shown that iron concentrations at the catalyst particle surface were significantly higher than the bulk iron concentrations for highly contaminated ECat-HIGH equilibrium catalysts. For example, surface enrichment ratio of iron for ECat-HIGH particles was 14 , i.e., iron concentration on the surface of ECatHIGH particle was 14 times higher than its bulk iron concentration. The surface enrichment ratios of iron for ECat-LOW and ECat-INT were found to be 1.8 and 2.7 respectively. Presence of iron in the form of $\mathrm{Fe}_{2} \mathrm{O}_{3}$ on the catalyst surface was inferred from the XPS binding energy of 710.6. Knowing that ECat-LOW is the youngest of all equilibrium catalysts, the higher iron concentration on the surface of ECat-HIGH compared to ECat-LOW and ECat-INT is a clear indication of iron accumulation on the catalyst surface as the catalyst ages. All of these findings are consistent with the previous investigations. Beyerlin et al. [2] have also confirmed the presence of high concentrations of iron at the periphery of the certain sections of FCC catalyst by using X-ray microscopy along with scanning electron microscopy. Their results have shown that iron, like nickel, also remains fixed at the catalyst particle surface.
Another interesting finding was to observe the FCC catalyst particles sticking to each other and particles having irregular shapes as seen from the SEM micrograph given in figure 5(A) and (C). The number of particles sticking to each other in highly contaminated ECat-HIGH catalyst sample was higher compared to ECat-LOW catalyst samples. The elemental analysis at the interface (marked area with a white rectangle in figure 5(B)) of sticking particles shows the significant amounts of iron at least higher than the iron amount detected in the regions away from the interface. This could be an evidence of the agglomeration of the catalyst particles due to iron contamination. However, we are still looking for a stronger evidence to support this claim.

Hodgson et al. [11] reported the severe impact of iron contamination on FCC unit performance. They introduced a test method called "Akzo Nobel Accessibility Index" that quantifies the catalyst accessibility. They claimed that the quantification of catalyst accessibility with this test method could be used to optimize the catalyst performance. They found that the formation of a crust over the surface of the catalyst particle caused the low catalyst accessibility by limiting the diffusion of hydrocarbons into the active sites. Formation of this iron crust also changes the catalyst surface features. These specific features on the catalyst surface may play an important role for hydrocarbon adsorption and cracking. However, a more detailed study by combining several techniques is needed to understand the effects of surface topography on the catalyst performance.

\section{Conclusions}

Deposits on the surface of equilibrium FCC catalysts have been visualized by means of AFM and SEM. Topography obtained by AFM and SEM was in good agreement. The chemical composition on the surface of catalyst particles was determined by using SEM-EDS. The elemental analyses have shown that these deposits contain iron. Our results indicate the accumulation of iron on the external catalyst surface as the catalyst ages. These deposits or nodules on the external surface of the catalyst particle may change the specific features on the catalyst surface and play an important role for hydrocarbon adsorption and cracking. AFM together with SEM-EDS could be used to determine the iron contamination of the FCC catalyst.

\section{Acknowledgments}

Support from the West Virginia University Department of Chemical Engineering is gratefully acknowledged. We acknowledge Mr. Howard Moore, Ashland Marathon LLC, for his helpful discussions and for providing the equilibrium FCC catalyst samples used in 

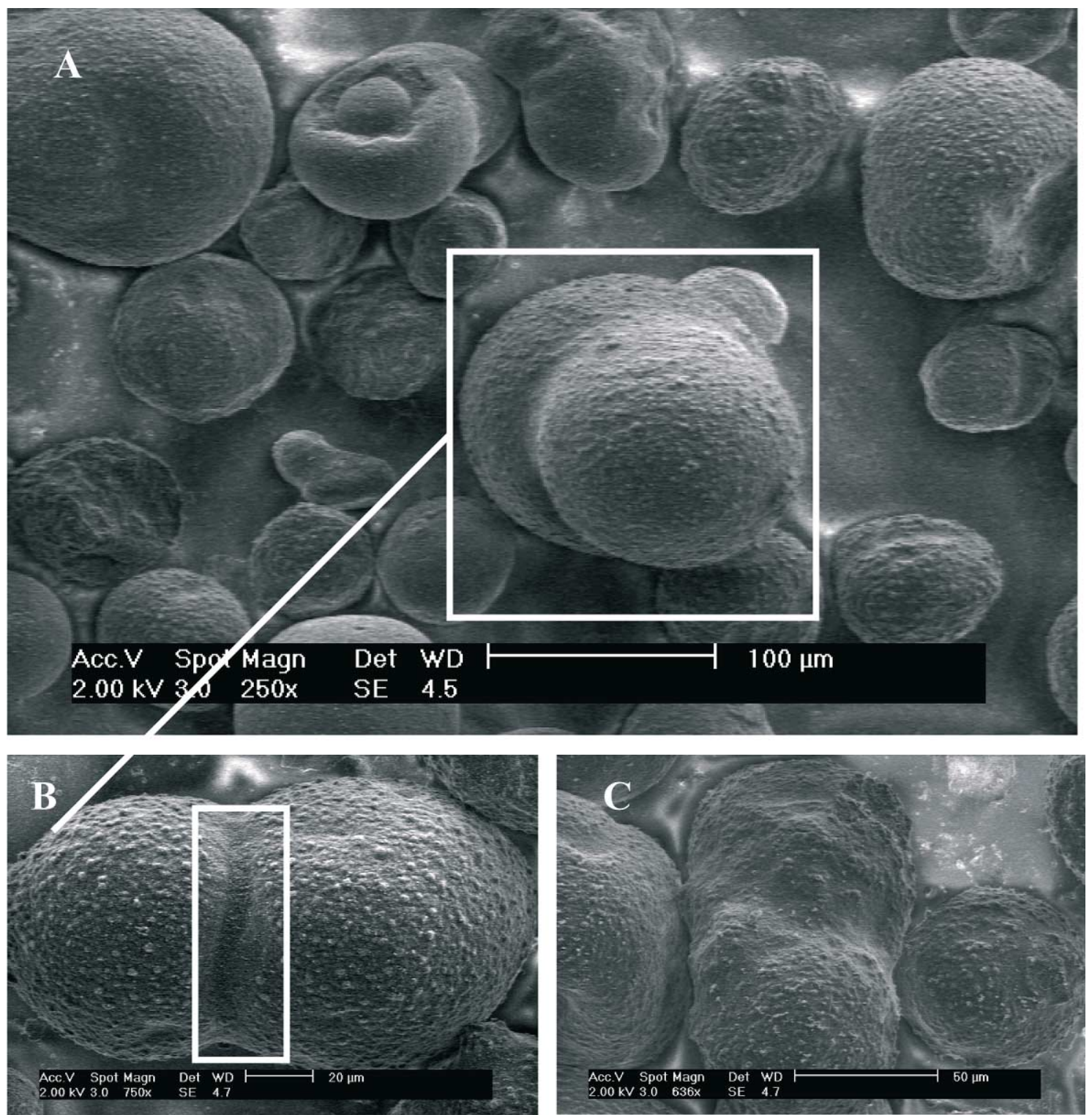

Figure 5. SEM images of ECat-HIGH: $(\mathrm{A}) \times 250$, (B) $\times 750$, and $(\mathrm{C}) \times 636$ magnifications.

this study. We would like to thank Mr. Vikram Tolani who helped in the operation of AFM equipment at the National Institute of Occupational Safety and Health (NIOSH), Morgantown, through the courtesy of Dr. William E. Wallace.

\section{References}

[1] P.B. Venuto and E.T. Habib, Catal. Rev. Sci. Eng. 18 (1978) 1.

[2] R.A. Beyerlein, W.J. Reagan, G.W. Zajac, J.B. Hall, B.J. Huggins, C. Choi-Feng, K.W. Jones and P. Spanne, 13th North American Meeting of the Catalysis Society (Pittsburgh, PA, 1993), Paper B-12.
[3] S.L. Suib, E.V.R. Borgstedt, H. Cao and M.L. Occelli, in Catalyst Deactivation, C.H. Bartholomew and J.B. Butt (eds) (Elsevier Science Publishers B.V., Amsterdam, 1991), pp. 717-722.

[4] E.L. Kugler and D.P. Leta, J. Catal. 109 (1988) 387.

[5] S. Jaras, Appl. Catal. 2 (1982) 207.

[6] M.L. Occelli, S.A.C. Gould and B. Drake, Microporous Mater. 2 (1994) 205.

[7] M.L. Occelli, S.A.C. Gould, J.M. Tsai and B. Drake, J. Mol. Catal. A: Chem. 100 (1995) 161

[8] M.L. Occelli, F.E. Huggins, J.M. Dominguez, J.M. Stencel and S.A.C. Gould, Microporous Mater. 4 (1995) 291.

[9] M.L. Occelli and S.A.C. Gould, Chemtech May (1994) 24

[10] M.L. Occelli, M. Kalwei, A. Wölker, H. Eckert, A. Auroux and S.A.C. Gould, J. Catal. 196 (2000) 134

[11] M.C.J. Hodgson, C.K. Looi and S.J. Yanik, Akzo Nobel Catalysts Symposium (Noordwijk, The Netherlands, 1998). 